\title{
New Technologies and New Paradigms in Historical Research
}

\author{
Antonio Cartelli \\ University of Cassino \\ Italy \\ eartan@offieine.it
}

\author{
Luisa Miglio \\ University "La Sapienza" \\ Italy
}

\author{
Marco Palma \\ University of Cassino \\ Italy \\ mpatma@umieas.it
}

\begin{abstract}
After a short introduction on media evolution and their implications on human history the paper presents the results of two experiences held by the authors while using new technologies in disseminating bibliographical and historical information.

The former experience concerns the Web publication of a bibliography on Beneventan manuscripts and arises from the need of overcoming the long edition times of printed information. It also proposes itself as an online resource for all researchers involved in studies on the South Italian book script in the Middle Ages. The latter one originates from most recent studies on women copyists in the Middle Ages and uses an online database to spread news on this subject.

The paper then analyzes analogies and differences between the two experiences and suggests, at last, they can be seen as a source of online information for scholars, thus representing a first step towards the construction of new paradigms of knowledge and research in historical studies.
\end{abstract}

Keywords: Data Base, Historical Research, Knowledge Paradigm, Middle Ages, Web.

\section{Introduction}

During this last century mass-media and multi-media communications underwent a progressive growth and a smart evolution, at such a point that they are today pre-eminent with respect to printed matter, not only in broadcasting news but also in propagating more structured and jointed information such as disciplinary knowledge and more general cultural matters.

Many scholars attribute the reasons of such a revolution to the structural characteristics of new media with respect to printed texts, because of their directness, effectiveness and immediateness, while transmitting all kinds of information, or, to repeat the same words used by D. de Kerckhove (de Kerckhove 1995) - these media talk to the body more than to the mind -, they wrap up information addressees with their messages.

Material published as part of this proceedings, either on-line or in print, is copyrighted by the author with permission granted to the publisher of Informing Science for this printing. Permission to make digital or paper copy of part or all of these works for personal or classroom use is granted without fee provided that the copies are not made or distributed for profit or commercial advantage AND that copies 1) bear this notice in full and 2) give the full citation on the first page. It is permissible to abstract these works so long as credit is given. To copy in all other cases or to republish or to post on a server or to redistribute to lists requires specific permission from the author.
The great changes induced by new media in human behaviour forced many researchers to carry out deep studies to analyze their influence on human history and evolution. It's important to remember here, for the implications they have in what follows, M. McLuhan's and D. de Kerckhove's contributions.

The former one is famous for at least two symbolic assertions characterizing his work: first of all he says that every instrument mankind created in the history can be thought of as a prosthesis, i.e. a tool to improve human abilities and to multiply the ways of understanding phenomena; then he asserts that the medium is the message, thus carrying to the extremes the influence communication media can have on people, much more than the contents of the messages themselves.

The latter one as a pupil and heir of McLuhan continued his researches and enriched his assertions with new contents.

A common opinion concerning the characteristics of the phase today's mankind (or at least a part of it) lies in seems to join many scholars (including the above ones). They point out the presence of media extending in space and time individual capabilities and skills, so annulling the answering time to external stimulation. If this is the end act of the human evolution it is perhaps too early to say but it's 


\section{A. Cartelli, L. Miglio, M. Palma}

undoubtedly a step of the human evolutionary route having no comparison with any other event until now. The importance of finding a key to understand this phenomenon leis in the following reasons: 1) the overcoming of the optimist-pessimist dualism (the former one supporting a total democracy governed by media, the latter one persuaded that new media will delete the ego and homologate the masses); 2) the influence of hyper-sensitization and overexposition to new media on future scenarios mankind will deal with.

In any case the roots of today events are very far: they start from language invention, pass through alphabet discovery and printing invention and arrive to today's new media.

Language is for de Kerkchove the first example of human software and the first tool human beings used to create, model and dominate matter, to share their experiences and to build more and more complex social structures (Calvani 1997).

Alphabet and writing, after the language, induced another revolution marked by two main aspects: on one side we can find the loss of some peculiarities of oral cultures such as concepts and ideas redundancy in communication processes, the dependence of human expression on memory and context etc.; on the other side they stimulated the birth of philosophical and scientific-logic thinking (many authors assert it's not a case the roots of western philosophy are Greek and Greek is also the first alphabet associating each character to a pronounceable sound [Ong 1986, Olson 1974]).

A further revolution in communication come from Gutenberg's invention of printing. It deleted the last traces of oral tradition in manuscript communication, made possible universal alphabetization and emphasized reading personalization (the potential diffusion of printed texts emphasized individual reading, usually silent, and the consequent re-elaboration of read matters).

Printed texts also imposed a different structure in information organization because of their shortage of social aspects with respect to manuscripts. The structure of printed books made impossible the modification of the original texts with glosses and notes in the margin; moreover as the books were designed for single readers they revealed "closed objects", i.e. it was now absolutely impossible to make changes on them or to transmit to other people eventual notes a reader could make on them.
Many authors, in the last decades, hypothesized a great influence of the invention of printing on the evolution of occidental culture because of the characteristics of printed texts. They particularly asserted that the linearity of printed texts, their ordered succession of sentences, their rigid division in paragraphs and chapters and their use of indexes led to the partition of knowledge in separated compartments with the consequence of a rigid disciplinary structure of knowledge.

The last revolution in human communication is attributed by many authors to the diffusion of electricity and electronics because of the dependence of new media on the instruments and the machines using these technologies. To be more precise one can say there isn't a unique point of view in this last phase of human history and many researches suggest at least two well distinct periods: the former one marked by the presence of mass-media, particularly the television, the latter one featured by computer presence in all human activities and its evolution/transformation from a computational machine into a data elaboration instrument and, at last, into a communication medium.

Computer use and human computer interaction changed during last decades due to the evolution of software applications, hypertext invention and GUI (Graphics User Interfaces) and the ways information were spread out changed consequently. The last step of the revolution characterizing computer evolution from data elaboration into pure communication is mostly attributed to computer networks and particularly to the Internet.

Many psychological and sociological studies have been carried out to determine the effects of computer use on human behaviour and on CMC (Computer Mediated Communication) and different hypotheses were proposed to explain the changes in attitudes and manners related to the use of networks in social contexts. Consequences of the above researches were, among the others, new community meanings, new values for the terms ethics and economy and the presence of a Network Cybercanalization, i.e. the presence of some little but very specialized communities made up by individuals connected via Internet, who often don't know each other, are living all over the world and share the same interests (Paccagnella, 2000). 


\section{Middle Ages and the Internet: Two experiences}

\section{The Bibliography of Beneventan Manu- scripts}

This experience began in 1991 within the School for Archivists and Librarians and the Department of Philology and History of the University of Cassino, where started the collection of the quotations concerning the manuscripts written in the medieval Southern Italy national script. Main purpose of the work was a yearly bulletin devoted to the studies on Beneventan manuscripts (for sake of completeness it's suitable to remember that the term "Beneventan script" denotes a specific writing style used in Southern Italy during the Middle Ages). Every quotation of a Beneventan manuscript in all publications from 1990 onwards is composed of the shelfmark (i. e. town, library and number) and a short abstract explaining the reason of the quotation.

Bibliographic materials were picked up by many contributors, most of which were students or ex-students of the School, and not exclusively Italian. They were helped by a software package, a special version of a computer program called BIBMAN used at ICCU (Istituto Centrale per il Catalogo Unico delle Biblioteche Italiane) for the bibliography of manuscripts kept in Italian Libraries. Every year up to 2000 a volume collecting all bibliographic materials was edited by the publisher Viella in Rome (AA. VV. 1993-).

Until the creation of the Web site concerning the bibliography of Beneventan manuscripts the diffusion of this kind of information was entrusted only to the printed volumes published once a year and every attempt of using floppy disks, or other computer media, to disseminate information with a different periodicity had quite no results. It has to be noted that the technique of floppy shipping was already suggested in the introduction to the first volume of the collection. Librarians, scholars and all users of the printed materials were given the possibility to create personal data bases from the whole set of data.

In accordance with the publisher a Web site was inaugurated in 1997 with the main aim of making faster and easier for researchers to download new data, approximately every month.

The main problem during the site creation was represented by the visualization within a Web page of the data pro- duced by the software BIBMAN, as these data were a text file in ASCII code. This file couldn't be replaced by any other kind of software material because of the good skills the contributors gained while using this software, so that the authors decided to produce two different versions of the same bibliographic sources. A former one consisted of a Web page with a directly readable version of the data, obtained by including in the HTML tags $<$ PRE $>$... $</$ PRE $>$ the ASCII code produced by the software BIBMAN; a latter one, a file that could be downloaded by simply clicking on a special button, was the same file obtained by BIBMAN and could be used by every reader owning the same program to create his own database.

From 1997 onwards other pages, together with the bibliographic data, have been added to the site, first of all a list of selected links to other sites, useful to find further bibliographic information concerning manuscripts, then an inventory of the shelfmarks of Beneventan manuscripts.

The site is today a powerful resource for Middle Ages scholars (Fig. 1 shows a picture of the home page) who can get fresh information on publications relating to literature, art, history, script (it is accessible on line at:
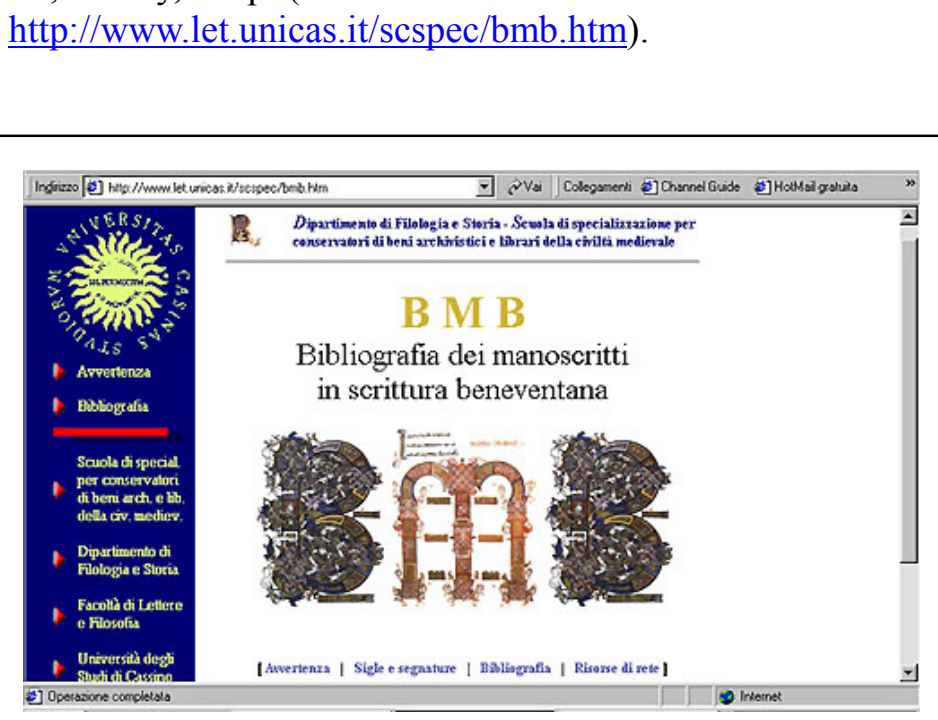

Fig. 1 - Home page of the BMB Web site

\section{Women Copyists in the Middle Ages}

It's not commonly known that women wrote manuscripts in the Middle Ages.

The new experience the authors are carrying out proposes an online database reporting all information on women 


\section{A. Cartelli, L. Miglio, M. Palma}

copyists leaving a trace of their work until the 15th century (in this first phase are excluded miniaturists and binders and every other person not directly involved in handwriting). The data appearing relevant for scientific community and further researches are:

- name - the name of the woman as it appears in manuscripts

- qualification - it is reported, if it is known, whether the copyist was a nun or a lay woman

- date - the date or the period the manuscripts belong to

- place - where the manuscripts were written

- country - the abbreviation of the country to which the place of origin belongs

- shelfmark - town, libray and number of the manuscripts

- text - authors and titles of the texts

- bibliography - the source of information about the manuscripts

The site, that will not give rise to a printed bulletin or other periodical printed matter on women copyists, has the following main characteristics:

1. it proposes itself as a continuous work-in-progress where everyone can suggest new data to include, by pointing out bibliographical information to the authors (in the site are reported their e-mail addresses);

2. it presents two separated sections: the former one can be operated only by the editors who can insert, modify, delete the stored data, thus ensuring the scientific validity of the information reported; the latter one is at everyone's disposal to obtain the list of all women in the database or to make queries concerning women with specific requisites (Fig. 2 shows the Home Page of the site);

3. the editors can enter the database without being

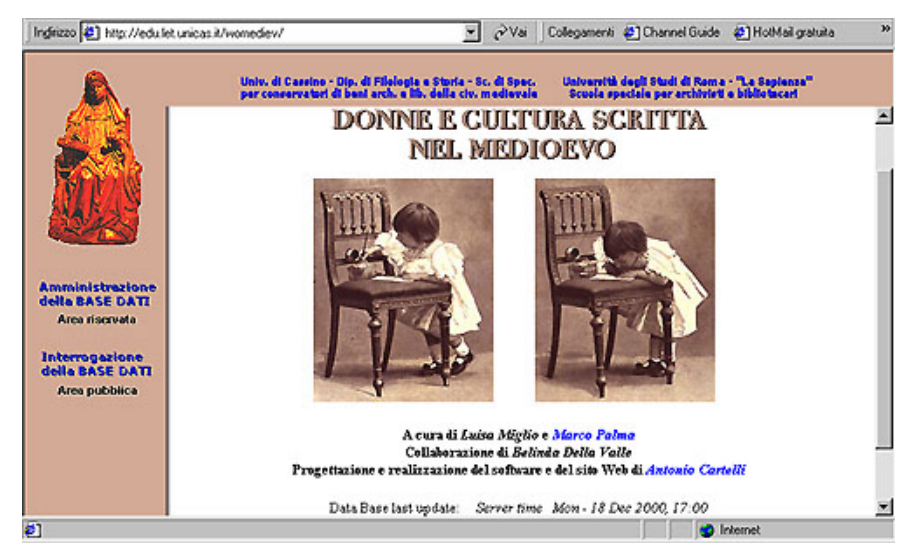

Fig. 2 - Home Page of the Women Copyists site obliged to physically sit at the computer the data base lays in; they can also use the database management services wherever they are all over the world on condition they can access Internet in some a way (by phone, by LAN etc.). The site is accessible on line at: http://edu.let.unicas.it/womediev/

A common characteristic of the above experiences is represented by the exclusive use of free or open source software that is: Linux as the Operating System, Apache as the Web server, Postgres SQL as the RDBMS and PHP-3 as the front end language for the data base.

\section{Towards new research paradigms}

At a first glance the experiences made up by the authors with the above Web sites on medieval themes, also if devoted to very specific themes appear very similar to what's being experienced elsewhere on the same or different arguments and seem to assert nothing particularly new in the context of the scientific debate on the use of the Web for spreading information. It's well known indeed that the Web is used by many institutions and for different reasons to publish information concerning the same or different arguments.

A few considerations follow in spite of what has just been said to show analogies and differences of these experiences and their consequences.

First of all both experiences use the Web as an instrument for spreading information on the Net with no difference among scholars or simple readers. Moreover if it's true the two sites are concerned with different arguments, Beneventan script the former and women copyists the latter, they together contribute in spreading news on the Middle Ages and propose themselves as an instrument for enforcing among researchers the feeling of belonging to a scientific community; i.e. they make real the cybercanalization among little, very specialized communities presented above.

While reading more carefully the description of the two experiences one can notice a basic difference between them: the former one was borne from a previous experience based on the publication of a book (the software BIBMAN storing the bibliographical data was used only to collect and catalogue more easily and quickly the same data) and the Web site had the main function of shortening the editing times of information leaving to the yearly published book the task of being the reference element in scientific knowledge. The latter one, on the other side, 


\section{New Technologies and New Paradigms}

proposes itself as a source of information only on the Web, thus imposing the revision of the common idea until now accepted by everyone that only printed matter can warrant the scientific validity of any kind of assertion.

Absoluteness and immutability of printed texts seem in fact to characterize most part of the Middle Ages scholars, also if not only them, and this preconception is probably born in a context where the influence of the Gutenberg medium was very strong. It's now required a great cultural change and probably a new knowledge paradigm.

The structure and the characteristics of this paradigm can be hypothesized after observing the influence of new media on the two forms of knowledge: the common one relying on everyday individual experience and the scientific one that can also be borrowed by different disciplinary contexts.

On the individual side some differences characterizing the communication on the Net with respect to printed matters can be summarized as follows:

1. on the Internet one cannot wait for the information to read but he has to search it (there is then the need for a cultural change in the user);

2. people looking for information on the Web must have a greater selecting skill with respect to people working with printed matter;

3. the hypertext structure of the Web imposes the user a continuous comparison of his structure and articulation of knowledge with respect to the information he is browsing;

4. every user while experimenting problems or troubles of any kind on the Net can resort to types of help online by using other communication services like email, chat or newsgroup.

The influence of the electronic medium on the construction of new knowledge seems more difficult to recognize and to describe from a theoretical point of view. It's well known indeed that the traditional scientific knowledge lies on qualitative and quantitative methods that are, to cite only some of them, the experimental one, the autobiographical one, the comparative one, the hermeneutical one etc.

The following questions arise then naturally: what kind of influence and what modifications can be induced by the electronic medium on the above methods? Is there the need for other knowledge methods?
It's in the authors' opinion that there are at least two different points of view answering to the above questions, the one opposite to the other: the former one seeing in the new technologies the instruments to simplify and make quick and fast the operations usually made by generations of researchers, thus adding nothing new to well consolidated methods and strategies; the latter one characterizing people with good skill in online communication techniques and practices thus seeing in the Net a prosthesis to acquire and construct a new scientific knowledge.

This last point of view is defined by the authors online knowledge and is proposed to the reader as a new knowledge paradigm to analyze in greater detail as follows.

First of all why a new definition? The authors are persuaded of the need of the new term owing to the different aspects other definitions dwell upon while analyzing communication-knowledge processes on the Net. For example de Kerchkove's collective intelligence (de Kerchkove 1998) gives reason of socialization and reelaboration phenomena that communication can activate; on the other side the term shared knowledge is used by other researchers to underline the function of communication channel evidenced by the Net in human communication.

A first concise but exhaustive attempt to define online knowledge is the following one: it's the process leading to new knowledge made up by previous knowledge by means of all synchronous and asynchronous communication services available on the Net. This knowledge method can be referred to theoretical and practical aspects in the same manner, as it can be useful in the interpretation of phenomena or the proposal of hypotheses, as well the design of hardware and/or software instruments.

Concrete and consolidated examples of this paradigm can be found in the experiences of Net communities working on software projects like the Apache group, the Linux Users Group or the GNU project.

The authors are persuaded that the experience they are leading goes in the same direction as the above ones and the women copyists site can be seen as a first attempt of application of the online knowledge paradigm to historical research. 


\section{Conclusion}

After a short description and analysis of the evolution of human communication processes the authors hypothesize the extension of an online knowledge paradigm to historical research and suggest that the experiences they are leading are going exactly in this direction.

An automatic extension of their hypothesis to all people involved in historical researches or to all possible fields of human knowledge is quite impossible because of the presence of serious troubles and doubts in the acquisition of these new paradigms by many scholars.

Is there a "human factor" influencing the use of new knowledge paradigms and particularly of the online knowledge one? According to the authors problems in the acquisition and use of this paradigm can be attributed to the firm belief evidenced by many people that printed matter is the only medium granting the survival of the results of their personal research. Perhaps the current debate on copyright, security and validation of Web material will provide scientific information with the guarantees scholars are expecting. But it is a long way to this goal.

\section{References}

AA., VV., (1993-). BMB. Bibliografia dei manoscritti in scrittura beneventana, , 1-, Roma: Viella.

Calvani, A. (1997). Dal libro stampato al libro multimediale, Scandicci (FI): La Nuova Italia.

de Kerckhove, D., (1995). The Skin of Culture, Toronto: Somerville House Books Ltd. de Kerckhove, D., (1998). Rischi pochi, vantaggi tanti: oggi è meglio studiare on line, Telèma, 12 . Accessible on line at: tttp://www.fub.it/telema/TELEMA12/DeKerc12.html

Olson, D. R. (1974). Media and Symbols. The Forms of Expression, Communication and Education, Chicago: University Press.

Ong, W. J. (1986). Oralità e scrittura, Bologna: Il Mulino.

Paccagnella, L. (2000). La comunicazione al computer, Bologna: Il Mulino.

\section{Biographies}

Antonio Cartelli is a teacher of CS (i.e. the European I.T.) in an Italian Technical High School and has a yearly contract for teaching Educational Technologies at the Faculty of Humanities in the University of Cassino - Italy. A. Cartelli is also working on various Network Projects the same Faculty started or has designed to build up.

Luisa Miglio is a faculty member of "La Sapienza" University in Rome - Italy. L. Miglio teaches Codicology at the School for Archivists and Librarians and the writing of women (particularly in the last centuries of the Middle Ages) has always been the center of her research activity.

Marco Palma is a faculty member of the University of Cassino - Italy, where he teaches Latin Paleography at the Faculty of Humanities. Currently he works on the material aspects of manuscripts as well the writing of books and charters in the Middle Ages. 